\title{
Erratum: Radiation emitted by transverse-gradient undulators [Phys. Rev. Accel. Beams 19, 090704 (2016)]
}

\author{
Axel Bernhard๑, Nils Braun, Verónica Afonso Rodríguez, Peter Peiffer, \\ Robert Rossmanith, Christina Widmann, and Michael Scheer
}

(Received 28 August 2020; published 28 September 2020)

DOI: 10.1103/PhysRevAccelBeams.23.099901

In the figures showing comparisons of on-axis photon flux per unit solid angle were labeled incorrectly. The dimensions should have read $1 /\left(\mathrm{s} \mathrm{rad}^{2} 0.1 \% \mathrm{bw}\right)$ instead of $1 /\left(\mathrm{s} \mathrm{mrad}^{2} 0.1 \% \mathrm{bw}\right)$. Consequently, the numbers shown in these figures were too large by a factor of $10^{6}$.
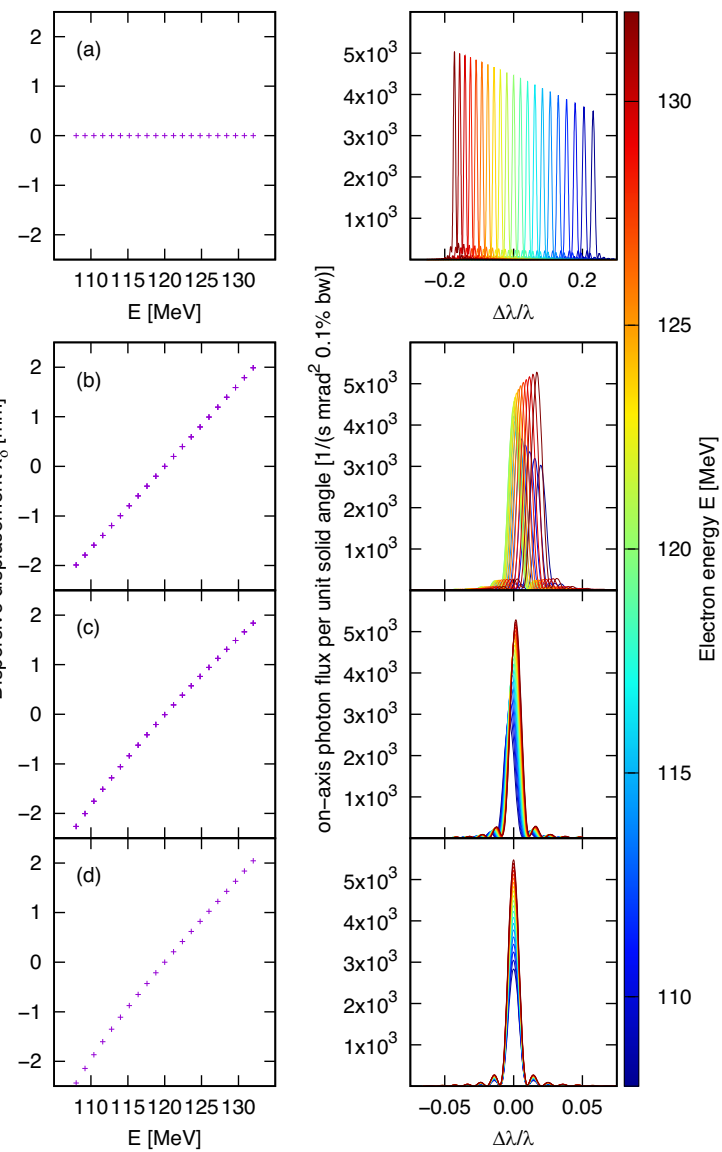

FIG. 5. Basic proof of the TGU concept: Electron energy dispersion and resulting radiation spectra for an electron energy band of $\Delta E=120 \mathrm{MeV} \pm 10 \%$ for the cases (a) planar undulator with $K=1.07$, (b) ideal linear TGU with $K_{0}=1.07$ and $\alpha=139.7 \mathrm{~m}^{-1}$ and linear dispersion (Eq. (4) of the original article), (c) the same with nonlinear dispersion according to Eq. (12) of the original article, (d) idealized cylindric SCTGU with optimized dispersion. For all cases a beam current of 10 pA, equally distributed over the 21 monoenergetic zero-emittance beamlets, is assumed.

Published by the American Physical Society under the terms of the Creative Commons Attribution 4.0 International license. Further distribution of this work must maintain attribution to the author(s) and the published articles title, journal citation, and DOI. 
Figures $5,6,7,8,11,12$, and 15 show the revised plots in now correct units of $1 /\left(\mathrm{s} \mathrm{mrad}^{2} 0.1 \% \mathrm{bw}\right)$. Since our arguments rely on comparisons of the quantities displayed in each of these plots, and all quantities which we compare to each other have to be corrected by the same factor, the conclusions of our article are not affected by this correction and remain unchanged.
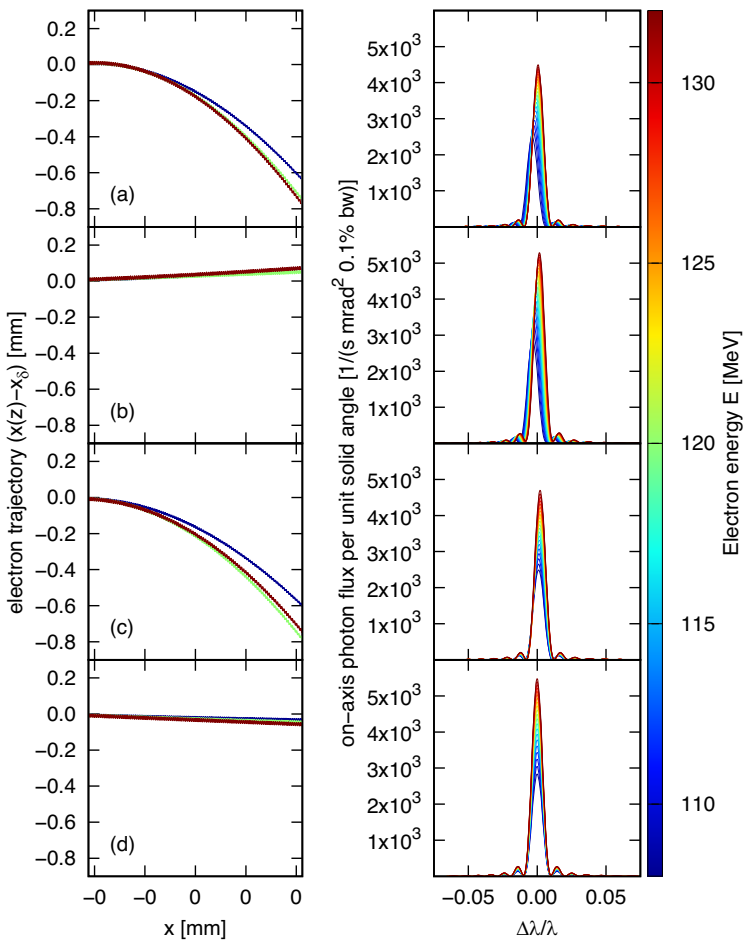

FIG. 6. The effect of the ponderomotive particle drift in TGUs: (a) linear TGU without trajectory correction and (b) with correction, (c) cylindric SCTGU without trajectory correction and (d) with correction.
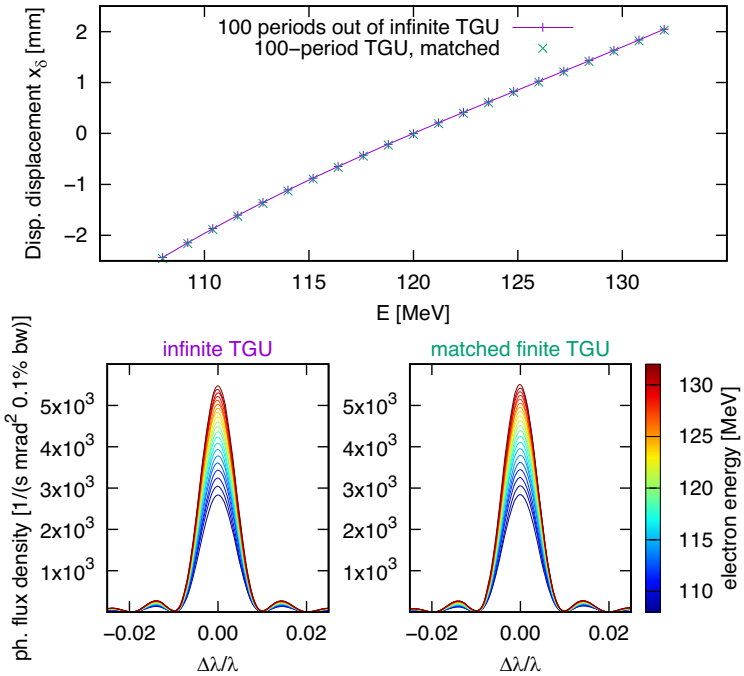

FIG. 7. Comparison of the required dispersion and the resulting radiation spectra (assuming zero-emittance beamlets) between the case of 100 periods out of an infinitely long SCTGU and the case of a finite 100-period SCTGU with matching periods. See the text for details. 

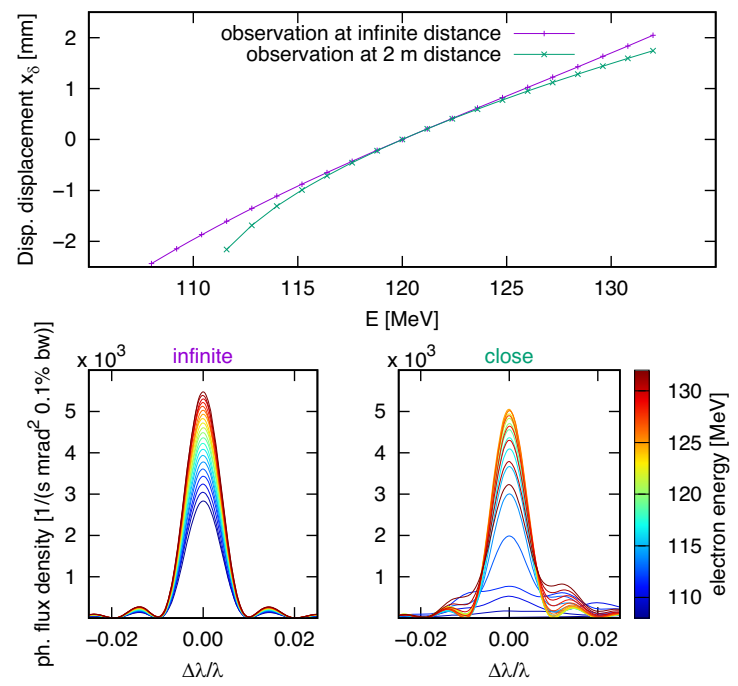

FIG. 8. Comparison of the optimized spectral dispersion of the electron beam and resulting radiation spectra for an observation point in infinite and $2 \mathrm{~m}$ distance, respectively.
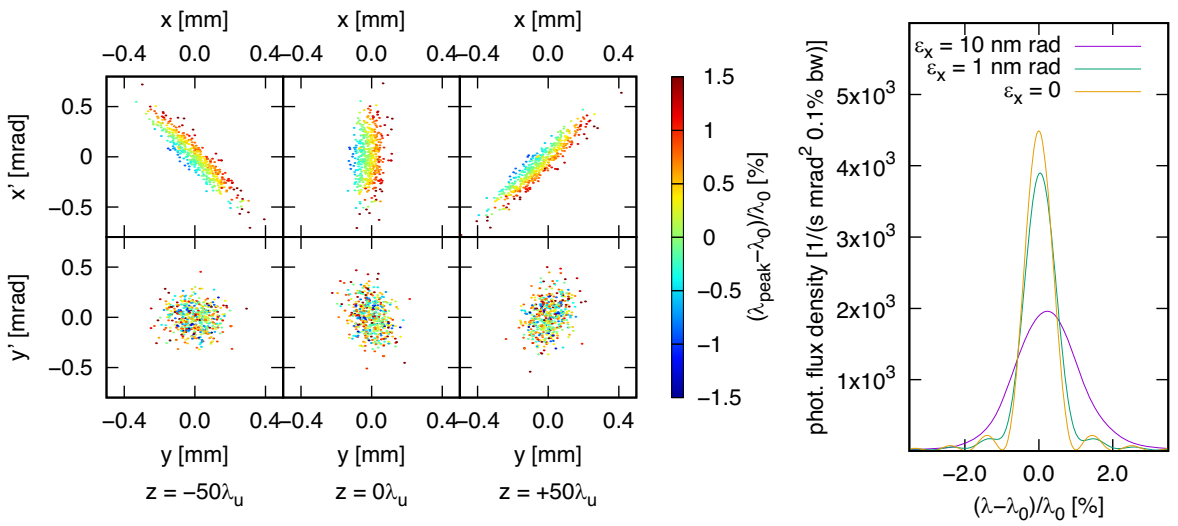

FIG. 11. Left: phase space distribution in $x$ and $y$ of a $120 \mathrm{MeV}$ electron bunch at the entrance, center and exit of the idealized cylindric TGU. The electron bunch parameters were chosen as $\beta_{x}^{\text {waist }}=0.5 \mathrm{~m}, \quad \beta_{y}=0.7 \mathrm{~m}=$ const and the geometric emittance $\epsilon_{x}=\epsilon_{y}=10^{-8} \mathrm{~m} \mathrm{rad}$. The color scale refers to the relative deviation of the peak energy of the radiation emitted by the respective particle with respect to that of the reference particle. Right: incoherently added radiation spectrum emitted by the particle bunch as a whole for different values of $\epsilon_{x, y}$, observed at infinite distance.

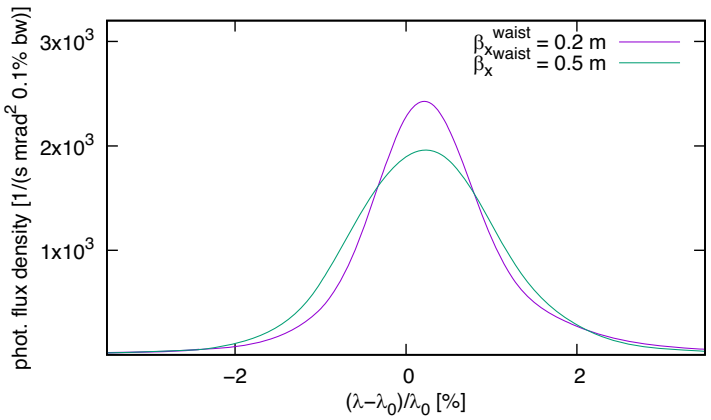

FIG. 12. Incoherently added radiation spectra emitted by particle bunches with $E=120 \mathrm{MeV}, \epsilon_{x}=10^{-8} \mathrm{~m}$ rad for two different choices of $\beta_{x}^{\text {waist }}$, observed at $10^{5} \mathrm{~m}$ distance. 


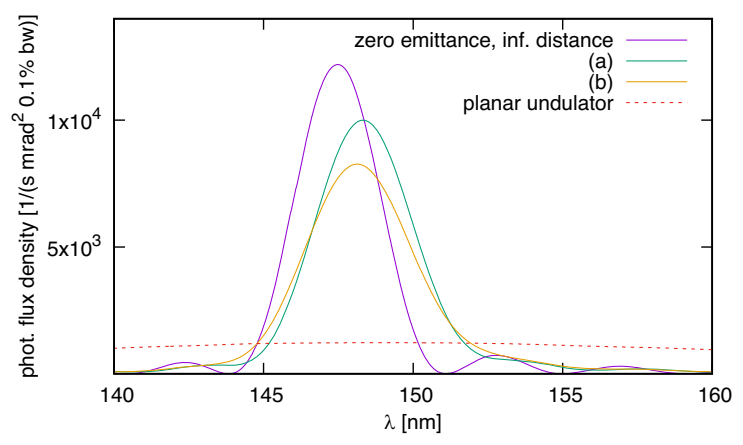

FIG. 15. Radiation spectra expected for the real SCTGU-P40 with finite beam emittance and observation at $2 \mathrm{~m}$ distance with (a) a photon optics for a parallel beam (zero angular acceptance) and (b) a point detector. The corresponding zero-emittance and planar undulator spectra in forward direction (parallel beam) are added for comparison. For the undulator and beam parameters refer to Table II of the original article.

The authors would like to thank the attentive PRAB reader who advised us of our mistake. 